\title{
Clusters of galaxies in the microwave band: Influence of the motion of the Solar System
}

\author{
J. Chluba ${ }^{1}$, G. Hütsi ${ }^{1}$, and R. A. Sunyaev ${ }^{1,2}$ \\ 1 Max-Planck-Institut für Astrophysik, Karl-Schwarzschild-Str. 1, 86740 Garching bei München, Germany \\ e-mail: [jchluba; gert] @mpa-garching .mpg . de \\ 2 Space Research Institute, Russian Academy of Sciences, Profsoyuznaya 84/32 Moscow, Russia
}

Received 2 September 2004 / Accepted 7 January 2005

\begin{abstract}
We consider the changes of the SZ cluster brightness, flux and number counts induced by the motion of the Solar System with respect to the frame defined by the cosmic microwave background (CMB). These changes are connected to the Doppler effect and aberration and exhibit a strong spectral and spatial dependence. The correction to the SZ cluster brightness and flux has an amplitude and spectral dependence, which is similar to the first order cluster peculiar velocity correction to the thermal SZ effect and hence may lead to a $\sim 10 \%$ correction to the kinetic SZ effect. Due to the change in the received cluster CMB flux, the motion of the Solar System induces a dipolar asymmetry in the observed number of clusters above a given flux level. Similar effects were discussed for $\gamma$-ray bursts and radio galaxies, but here, due to the very peculiar frequencydependence of the thermal SZ effect, the number of observed clusters in one direction of the sky can be both decreased or increased depending on the frequency band.
\end{abstract}

Key words. galaxies: clusters: general - cosmology: cosmic microwave background - cosmology: observations

\section{Introduction}

Due to the thermal SZ effect (Sunyaev \& Zeldovich 1972, 1980a) clusters of galaxies are (after our own Galaxy) one of the most important and brightest foreground sources for CMB experiments devoted to studying the primordial temperature anisotropies. Given the strong and very peculiar frequencydependence of the SZ signature (the flux changes sign at $v \sim$ $217 \mathrm{GHz}$ ), it is possible to extract these sources and thereby open the way for deeper investigations of the primordial temperature anisotropies, which for $l \gtrsim 3000$ are weaker than the fluctuations due to clusters of galaxies. Within the next 5 years, several CMB experiments like ACBAR, SZA, PLANCK, SPT, ACT, APEX, AMI and QUIET will perform deep searches for clusters with sensitivity limits at the level of $1-10 \mathrm{mJy}$ and in the future CMB missions such as CMBPOL should reach sensitivities 20-100 times better than those of PLANCK by using currently existing technology (Church 2002). Many tens of thousands of clusters will be detected allowing us to carry out detailed studies of cluster physics and to place constraints on parameters of the Universe like the Hubble parameter and the baryonic matter, dark matter and dark energy content (for reviews see Sunyaev \& Zeldovich 1981; Rephaeli 1995a; Birkinshaw 1999; Carlstrom et al. 2002).

Several groups have derived relativistic corrections to the thermal (Sunyaev \& Zeldovich 1972) and kinetic SZ (Sunyaev \& Zeldovich 1980b) effect as series expansions in the dimensionless electron temperature, $k T_{\mathrm{e}} / m_{\mathrm{e}} c^{2}$, and the cluster peculiar velocity, $\beta_{\mathrm{c}}=v_{\mathrm{c}} / c$ (Rephaeli $1995 \mathrm{~b}$; Challinor \& Lasenby 1998; Itoh et al. 1998a,b; Sazonov \& Sunyaev 1998).

Motivated by the rapid developments in CMB technology, the purpose of this paper is to take into account the changes in the SZ signal that are induced by the motion of the Solar System relative to the CMB rest frame. Assuming that the observed CMB dipole is fully motion-induced, this implies that the Solar System is moving with a velocity of $\beta_{\mathrm{o}}=v_{\mathrm{o}} / c=1.241 \times 10^{-3}$ towards the direction $(l, b)=$ $\left(264.14^{\circ} \pm 0.15^{\circ}, 48.26^{\circ} \pm 0.15^{\circ}\right)$ (Smoot et al. 1977; Strukov et al. 1987; Fixsen et al. 1996). As will be shown here, in the lowest order of $\beta_{\mathrm{o}}$ the motion-induced correction to the thermal SZ effect (th-SZ) exhibits an amplitude and spectral dependence, which is similar to the first order $\beta_{\mathrm{c}}$ correction to the th-SZ, i.e. the SZ signal $\propto \tau \beta_{\mathrm{c}} k T_{\mathrm{e}} / m_{\mathrm{e}} c^{2}$, where $\tau$ is the Thomson optical depth. On the other hand the observer's frame transformation of the kinetic SZ effect (k-SZ) leads to a much smaller y-type spectral distortion with effective $y$-parameter $\propto \tau \beta_{\mathrm{c}} \beta_{\mathrm{o}}$. Since both the amplitude and direction of the motion of the Solar System are well known the corrections we are discussing here are fully determined and can be easily taken into account in the process of estimation of the key cluster parameters.

Below we will consider two cases (i) when future multifrequency CMB experiments will be able to resolve clusters of galaxies; and (ii) when their angular resolution will not allow 
study of the clusters structures in detail. Therefore here we are not only discussing the change in the brightness of the CMB in the direction of a cluster but also the corrections to the flux as measured for unresolved clusters due to both the motioninduced change of surface brightness and the apparent change of their angular dimension. All these changes are connected to the Doppler effect and aberration, which also influence the primordial temperature fluctuations and polarization as discussed by Challinor \& van Leeuwen (2002).

Another important consequence of the motion of the Solar System with respect to the CMB rest frame is a dipolar anisotropy induced in the deep number counts of sources. This effect was discussed earlier in connection with the distribution of $\gamma$-ray bursts (Maoz 1994; Scharf et al. 1995) - identical to the Compton-Getting effect (Compton \& Getting 1935) for cosmic rays - and radio and IR sources (Ellis \& Baldwin 1984; Baleisis et al. 1998; Blake \& Wall 2002). The motioninduced change in the source number counts strongly depends on the slope of the $\log N-\log F$ curve and the spectral index of the source (Ellis \& Baldwin 1984), which makes it possible to distinguish the signals arising from different astrophysical populations. Here we show that a similar effect arises for the number counts of SZ clusters. Due to the very peculiar frequency-dependence of the th-SZ, the number of observed clusters in a direction of the sky can be either decreased or increased depending on the frequency band.

\section{General transformation laws}

A photon of frequency $v$ propagating along the direction $\boldsymbol{n}=$ $(\phi, \theta)$ in the CMB rest frame $S$ due to Doppler boosting and aberration is received at a frequency $v^{\prime}$ in the direction $\boldsymbol{n}^{\prime}=$ $\left(\phi, \theta^{\prime}\right)$ by an observer moving with the velocity $\beta_{\mathrm{o}}=v_{\mathrm{o}} / c$ along the $z$-axis:

$$
v=\gamma v^{\prime}\left(1-\beta_{\mathrm{o}} \mu^{\prime}\right) \quad \mu=\frac{\mu^{\prime}-\beta_{\mathrm{o}}}{1-\beta_{\mathrm{o}} \mu^{\prime}} .
$$

Here $\gamma=1 / \sqrt{1-\beta_{\mathrm{o}}^{2}}$ is the Lorentz factor, $\mu=\cos \theta$ and all the primed quantities ${ }^{1}$ denote the corresponding variables in the observer's frame $S^{\prime}$. It was also assumed that the $z^{\prime}$-axis is aligned with the direction of the motion. For a given spatial and spectral distribution of photons in $S$, in lowest order of $\beta_{\text {o }}$ the Doppler effect leads to spectral distortions, whereas due to aberration the signal on the sky is only redistributed.

\section{Transformation of the spectral intensity}

The transformation of the spectral intensity (or equivalently the surface brightness) $I(v, \boldsymbol{n})$ at frequency $v$ and in the direction $\boldsymbol{n}$ on the sky into the frame $S^{\prime}$ can be performed using the invariance properties of the occupation number, $n(v, \boldsymbol{n})=I(v, \boldsymbol{n}) / v^{3}$ :

$I^{\prime}\left(v^{\prime}, \boldsymbol{n}^{\prime}\right)=\frac{v^{\prime 3}}{v^{3}} I(v, \boldsymbol{n})$

\footnotetext{
${ }^{1}$ In the following, prime denotes that the corresponding quantity is given in the rest frame of the moving observer.
}

Here $I^{\prime}\left(v^{\prime}, \boldsymbol{n}^{\prime}\right)$ is the spectral intensity at frequency $v^{\prime}$ in the direction $\boldsymbol{n}^{\prime}$ as given in the rest frame of the observer. In the lowest order of $\beta_{\mathrm{o}}$ it is possible to separate the effects of Doppler boosting and aberration:

$I^{\prime}\left(v^{\prime}, \boldsymbol{n}^{\prime}\right) \approx I\left(v^{\prime}, \boldsymbol{n}^{\prime}\right)+\Delta I_{\mathrm{D}}\left(v^{\prime}, \boldsymbol{n}^{\prime}\right)+\Delta I_{\mathrm{A}}\left(v^{\prime}, \boldsymbol{n}^{\prime}\right)$.

with the Doppler and aberration correction

$\Delta I_{\mathrm{D}}^{\prime}\left(v^{\prime}, \boldsymbol{n}^{\prime}\right) \approx \beta_{\mathrm{o}} \mu^{\prime}\left[3-v^{\prime} \partial_{v^{\prime}}\right] I\left(v^{\prime}, \boldsymbol{n}^{\prime}\right)$

$\Delta I_{\mathrm{A}}^{\prime}\left(v^{\prime}, \boldsymbol{n}^{\prime}\right) \approx \beta_{\mathrm{o}} \sqrt{1-\mu^{\prime 2}} \partial_{\theta^{\prime}} I\left(v^{\prime}, \boldsymbol{n}^{\prime}\right)$.

Equation (3b) only includes the effects due to Doppler boosting, whereas Eq. (3c) arises solely due to aberration.

With Eq. (3) it becomes clear that in the first order of $\beta_{\mathrm{o}}$ any maximum or minimum of the intensity distribution on the sky will suffer only from Doppler boosting. This implies that due to aberration the positions of the central regions of clusters of galaxies will only be redistributed on the sky: in the direction of the motion clusters will appear to be closer to each other while in the opposite direction their angular separation will seem to be bigger. Another consequence of the observer's motion is that a cluster with angular extension $\Delta \ll 1$ in $S$ will appear to have a size $\Delta^{\prime}=\Delta\left[1-\beta_{\mathrm{o}, \|}\right]$ in the observer's frame $S^{\prime}$. Here $\beta_{\mathrm{o}, \|}=\beta_{\mathrm{o}} \mu^{\prime}$ is the line of sight component of the Solar System's peculiar velocity. Therefore in $S^{\prime}$ a cluster will look smaller by a factor of $1-\beta_{0}$ in the direction of the motion and bigger by $1+\beta_{\mathrm{o}}$ in the opposite direction. This implies that in the direction of the observer's motion, cluster profiles will seem to be a little steeper and more concentrated.

\section{Transformation of the measured flux}

The spectral flux $F\left(v, \boldsymbol{n}_{0}\right)$ from a solid angle area $\mathcal{A}$ in the direction $\boldsymbol{n}_{0}$ on the sky in $S$ is given by the integral $F\left(v, \boldsymbol{n}_{0}\right)=$ $\int_{\mathcal{A}} I(v, \boldsymbol{n}) \mu_{0} \mathrm{~d} \Omega$, where we defined $\mu_{0}=\boldsymbol{n} \cdot \boldsymbol{n}_{0}$. If one assumes that the angular dimension of $\mathcal{A}$ is small, then using Eq. (3) in the observer's frame $S^{\prime}$ the change of the flux due to Doppler boosting and aberration is given by

$$
\begin{aligned}
& \Delta F_{\mathrm{D}}^{\prime}\left(v^{\prime}, \boldsymbol{n}_{0}^{\prime}\right) \approx \beta_{\mathrm{o}, \|}\left[3-v^{\prime} \partial_{v^{\prime}}\right] F\left(v^{\prime}, \boldsymbol{n}_{0}^{\prime}\right) \\
& \Delta F_{\mathrm{A}}^{\prime}\left(v^{\prime}, \boldsymbol{n}_{0}^{\prime}\right) \approx \beta_{\mathrm{o}, \|} \int_{\mathcal{A}} \theta^{\prime 2} \partial_{\theta^{\prime}} I\left(v^{\prime}, \boldsymbol{n}^{\prime}\right) \mathrm{d} \theta^{\prime} \mathrm{d} \phi^{\prime},
\end{aligned}
$$

respectively. Assuming that the area $\mathcal{A}$ contains an unresolved object, that contributes most of the total flux and vanishes at the boundaries of the region, then the term arising due to aberration only can be rewritten as

$$
\Delta F_{\mathrm{A}}^{\prime}\left(v^{\prime}, \boldsymbol{n}_{0}^{\prime}\right)=-2 \beta_{\mathrm{o}, \|} F\left(v^{\prime}, \boldsymbol{n}_{0}^{\prime}\right) .
$$

This can be understood considering that in the direction of the motion the solid angle covered by an object is smaller by a factor $\left[1-\beta_{\mathrm{o}, \|}\right]^{2} \approx 1-2 \beta_{\mathrm{o}, \|}$. In this case the total change in the spectral flux $F^{\prime}\left(v^{\prime}, \boldsymbol{n}_{0}^{\prime}\right)$ is

$$
\frac{\Delta F}{F}=\frac{F^{\prime}\left(v^{\prime}, \boldsymbol{n}_{0}^{\prime}\right)-F\left(v^{\prime}, \boldsymbol{n}_{0}^{\prime}\right)}{F\left(v^{\prime}, \boldsymbol{n}_{0}^{\prime}\right)}=\beta_{\mathrm{o}, \|}\left[1-\frac{\partial \ln F}{\partial \ln v^{\prime}}\right] .
$$



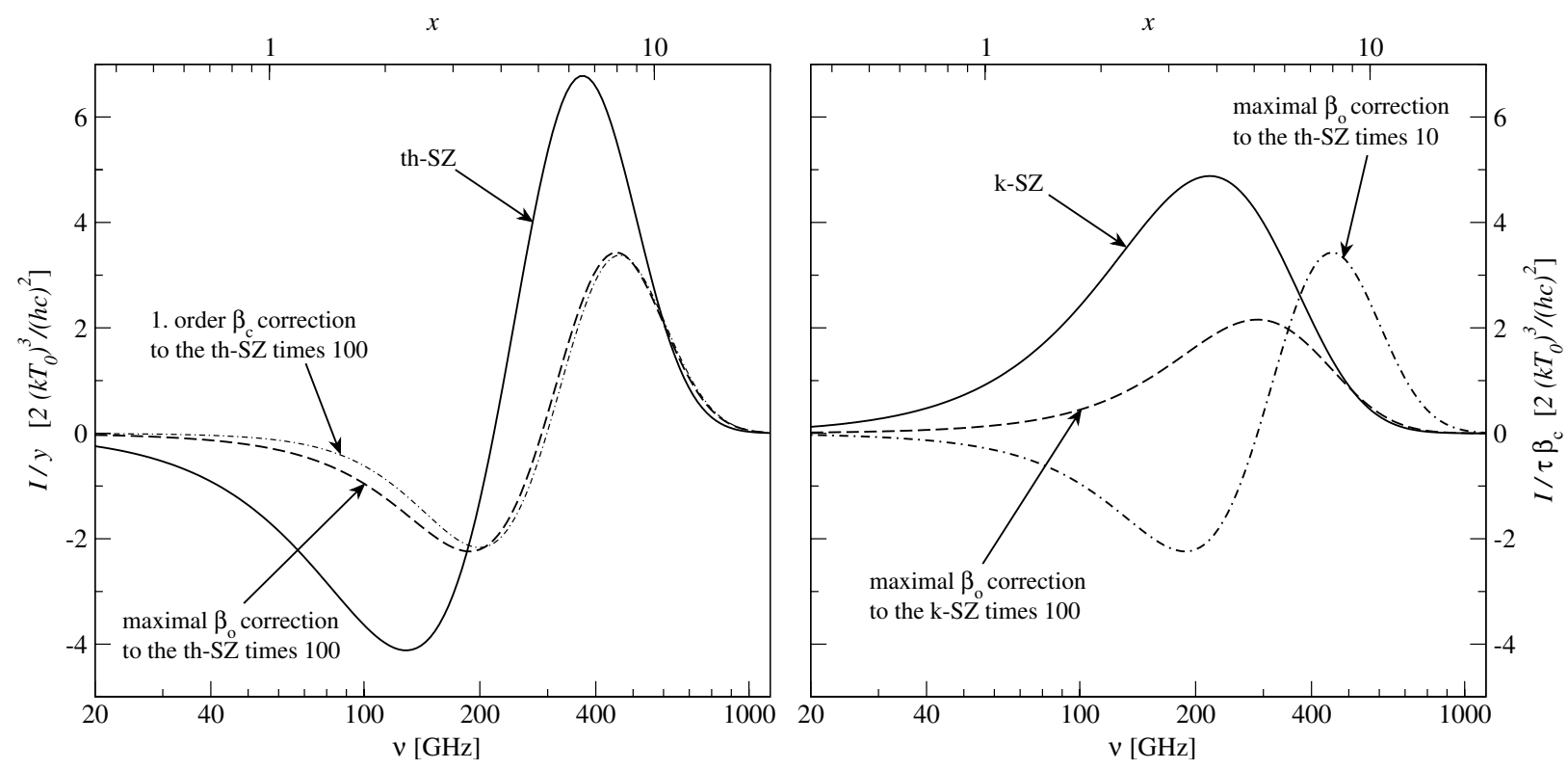

Fig. 1. Frequency dependence of the SZ brightness due to the non-relativistic th- and k-SZ and the corresponding corrections induced by the motion of the Solar System relative to the CMB rest frame for a cluster with electron temperature $k T_{\mathrm{e}}=5.1 \mathrm{keV}$, which is moving with $\beta_{\mathrm{c}}=10^{-3}$ towards the observer and is located at the maximum of the CMB dipole, i.e. $\beta_{\mathrm{o}, \|}=1.241 \times 10^{-3}$. The first order $\beta_{\mathrm{c}}$ correction to the th-SZ (dash-dotted line in the left panel) is shown. Note that for convenience all the velocity corrections are multiplied by a factor as given in the labels.

Integrating the flux $F^{\prime}\left(v^{\prime}, \boldsymbol{n}_{0}^{\prime}\right)$ over frequency $v^{\prime}$ it is straightforward to obtain the change of the total bolometric flux $F_{\text {bol }}^{\prime}\left(\boldsymbol{n}_{0}^{\prime}\right)=\int F^{\prime}\left(v^{\prime}, \boldsymbol{n}_{0}^{\prime}\right) \mathrm{d} v^{\prime}$ in the observer's frame $S^{\prime}$ :

$\frac{\Delta F_{\text {bol }}}{F_{\text {bol }}}=\frac{F_{\text {bol }}^{\prime}\left(\boldsymbol{n}_{0}^{\prime}\right)-F_{\text {bol }}\left(\boldsymbol{n}_{0}^{\prime}\right)}{F_{\text {bol }}\left(\boldsymbol{n}_{0}^{\prime}\right)}=2 \beta_{\text {o,ll }}$.

This result can also be easily understood considering the transformation law for the total bolometric intensity $I_{\mathrm{bol}}=\int I(v) \mathrm{d} v$, i.e. $I_{\mathrm{bol}}^{\prime}=I_{\mathrm{bol}} /\left[\gamma\left(1-\beta_{\mathrm{o}} \mu^{\prime}\right)\right]^{4}$, and the transformation of the solid angle $\mathrm{d} \Omega^{\prime}=\left[\gamma\left(1-\beta_{\mathrm{o}} \mu^{\prime}\right)\right]^{2} \mathrm{~d} \Omega$.

\section{Transformation of the number counts}

Defining $\frac{\mathrm{d} N}{\mathrm{~d} \Omega}(F, \boldsymbol{n})$ as the number of objects per solid angle $\mathrm{d} \Omega$ above a given flux $F$ at some fixed frequency $v$ and in some direction $\boldsymbol{n}$ on the sky in the CMB rest frame $S$, the corresponding quantity in the observer's frame $S^{\prime}$ may be written as

$\frac{\mathrm{d} N^{\prime}}{\mathrm{d} \Omega^{\prime}}\left(F^{\prime}, \boldsymbol{n}^{\prime}\right)=\frac{\mathrm{d} N}{\mathrm{~d} \Omega}(F, \boldsymbol{n}) \frac{\mathrm{d} \Omega}{\mathrm{d} \Omega^{\prime}}$,

where $F$ and $\boldsymbol{n}$ are functions of $F^{\prime}$ and $\boldsymbol{n}^{\prime}$. Now, assuming isotropy in $S$, in the first order of $\beta_{\mathrm{o}, \|}$ one may write

$\frac{\mathrm{d} N^{\prime}}{\mathrm{d} \Omega^{\prime}}\left(F^{\prime}, \boldsymbol{n}^{\prime}\right) \approx \frac{\mathrm{d} N}{\mathrm{~d} \Omega}\left(F^{\prime}\right) \times\left[1+2 \beta_{\mathrm{o}, \|}-\frac{\Delta F}{F} \frac{\partial \ln \frac{\mathrm{d} N}{\mathrm{~d} \Omega}\left(F^{\prime}\right)}{\partial \ln F^{\prime}}\right]$,

with $\Delta F=F^{\prime}-F$. For unresolved objects $\Delta F / F$ is given by Eq. (6). Here we made use of the transformation law for the solid angles and performed a series expansion of $\frac{\mathrm{d} N}{\mathrm{~d} \Omega}(F)$ around $F^{\prime}$.

If one assumes $\frac{\mathrm{d} N}{\mathrm{~d} \Omega}(F) \propto F^{-\lambda}$ and $F(v) \propto v^{-\alpha}$, it is straightforward to show that for unresolved sources $\frac{\mathrm{d} N^{\prime}}{\mathrm{d} \Omega^{\prime}}\left(F^{\prime}, \boldsymbol{n}^{\prime}\right) \approx$ $\frac{\mathrm{d} N}{\mathrm{~d} \Omega}\left(F^{\prime}\right)\left[1+\beta_{\mathrm{o}, \|}(2+\lambda[1+\alpha])\right]$. This result was obtained earlier by Ellis \& Baldwin (1984) for the change of the radio source number counts due to the motion of the observer. Depending on the sign of the quantity $\Sigma=2+\lambda[1+\alpha]$ there is an increase or decrease in the number counts in a given direction. However, in the case of clusters, $\alpha$ is a strong function of frequency, which makes the situation more complicated.

\section{Transformation of the cluster signal}

For an observer at rest in the frame $S$ defined by the CMB the change of the surface brightness in the direction $\boldsymbol{n}$ towards a cluster of galaxies is given by the sum of the signals due to the th-SZ, $I_{\text {th }}(v, \boldsymbol{n})$ and the $\mathrm{k}-\mathrm{SZ}, I_{\mathrm{k}}(v, \boldsymbol{n})$ :

$I_{\mathrm{SZ}}(v, \boldsymbol{n})=I_{\mathrm{th}}(v, \boldsymbol{n})+I_{\mathrm{k}}(v, \boldsymbol{n})$.

In the non-relativistic case these contributions are (see Zeldovich \& Sunyaev 1969; Sunyaev \& Zeldovich 1980a,b):

$$
\begin{aligned}
& I_{\mathrm{th}}(v, \boldsymbol{n})=y \frac{x \mathrm{e}^{x}}{\mathrm{e}^{x}-1}\left[x \frac{\mathrm{e}^{x}+1}{\mathrm{e}^{x}-1}-4\right] I_{0}(v) \\
& I_{\mathrm{k}}(v, \boldsymbol{n})=\tau \beta_{\mathrm{c}, \|} \frac{x \mathrm{e}^{x}}{\mathrm{e}^{x}-1} I_{0}(v),
\end{aligned}
$$

where $I_{0}(v)=\frac{2 h}{c^{2}} \frac{v^{3}}{\mathrm{e}^{x}-1}$ denotes the CMB monopole intensity with temperature $T_{0}=2.725 \mathrm{~K}, y=\int \frac{k T_{\mathrm{e}}}{m_{\mathrm{e}} c^{2}} n_{\mathrm{e}} \sigma_{\mathrm{T}} \mathrm{d} l$ is the Compton $y$-parameter, with the electron number density $n_{\mathrm{e}}$. In addition $\beta_{\mathrm{c}, \|}$ is the line of sight component of the cluster peculiar velocity and we introduced the abbreviation $x=h v / k T_{0}$ for the dimensionless frequency. Here we are only interested in the correction to the intensity in the central region of the cluster, where the spatial derivative of $y$ is small and the effects of 

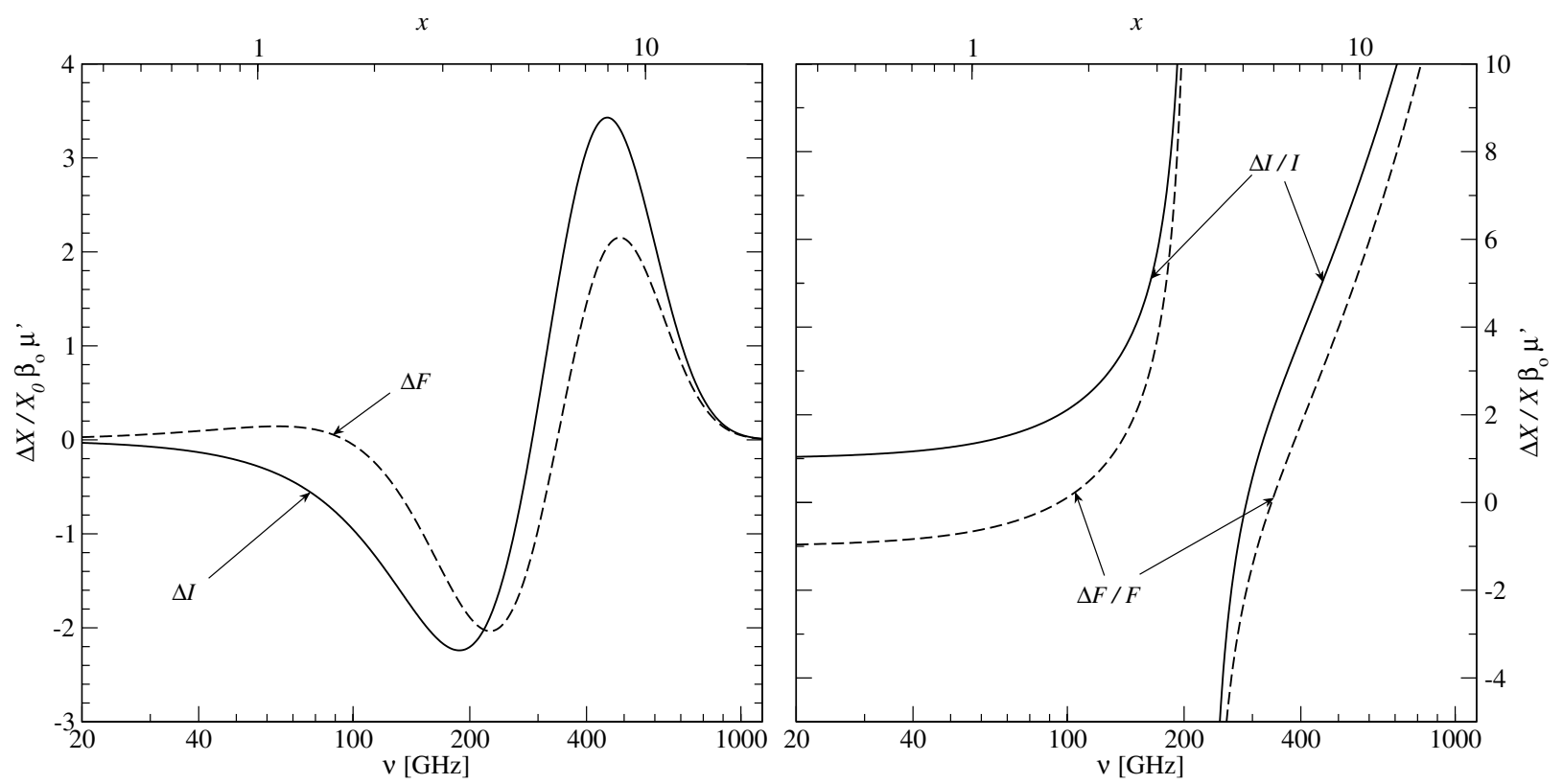

Fig. 2. Motion-induced change of the CMB spectral brightness $\Delta I$ and flux $\Delta F$ for a cluster of galaxies at rest with respect to the CMB. Left panel: absolute change of the cluster brightness (solid line) with $X_{0}=2.70 \times 10^{11} \mathrm{ymJy} / \mathrm{sr}$ and the flux for an unresolved cluster (dashed line) with $X_{0}=7.17\left\langle y / 10^{-4} r_{\mathrm{c}}^{2}\right\rangle_{\mathrm{cl}}\left(r_{\mathrm{c}} / 30^{\prime \prime}\right)^{2} \mathrm{mJy}$, where $r_{\mathrm{c}}$ is the core radius and where $\langle u\rangle_{\mathrm{cl}}=\int_{\mathrm{cl}} u \mathrm{~d} \Omega / 4 \pi$ denotes the cluster average of the quantity $u$. Right panel: relative change of the brightness (solid line) and flux for an unresolved cluster (dashed line). Here $\Delta I / I=\beta_{\mathrm{o}} \mu^{\prime}(3+\alpha)$ and $\Delta F / F=\beta_{\mathrm{o}} \mu^{\prime}(1+\alpha)$ (cf. Eq. (15)).

aberration may be neglected. Using Eqs. (3b) and (11) one may find

$$
\begin{aligned}
& \Delta I_{\mathrm{D}, \mathrm{th}}^{\prime}\left(v^{\prime}, \boldsymbol{n}^{\prime}\right)=y I_{0}\left(v^{\prime}\right) \frac{x^{\prime} \mathrm{e}^{x^{\prime}}}{\mathrm{e}^{x^{\prime}}-1} C_{\mathrm{th}}\left(x^{\prime}\right) \cdot \beta_{\mathrm{o}} \mu^{\prime} \\
& \Delta I_{\mathrm{D}, \mathrm{k}}^{\prime}\left(v^{\prime}, \boldsymbol{n}^{\prime}\right)=\tau \beta_{\mathrm{c}, \|} I_{0}\left(v^{\prime}\right) \frac{x^{\prime} \mathrm{e}^{x^{\prime}}}{\mathrm{e}^{x^{\prime}}-1} C_{\mathrm{k}}\left(x^{\prime}\right) \cdot \beta_{\mathrm{o}} \mu^{\prime}
\end{aligned}
$$

for motion-induced change of the cluster brightness. Here the functions $C_{\mathrm{th}}(\hat{x})$ and $C_{\mathrm{k}}(\hat{x})$ are defined by

$$
\begin{aligned}
C_{\mathrm{th}}(\hat{x}) & =4-6 \mathcal{X}+\mathcal{X}^{2}+\frac{1}{2} \mathcal{S}^{2} \\
C_{\mathrm{k}}(\hat{x}) & =\mathcal{X}-1,
\end{aligned}
$$

with the notations $\mathcal{X}=x \operatorname{coth}\left(\frac{x}{2}\right)$ and $\mathcal{S}=x / \sinh \left(\frac{x}{2}\right)$.

In Fig. 1 the spectral dependence of $\Delta I_{\mathrm{D}, \mathrm{th}}^{\prime}$ and $\Delta I_{\mathrm{D}, \mathrm{k}}^{\prime}$ is illustrated. The transformation of the th-SZ leads to a spectral distortion which is very similar to the first order $\beta_{\mathrm{c}}$ correction to the th-SZ. In the Rayleigh-Jeans limit $C_{\mathrm{th}}(\hat{x}) \rightarrow-2$ and therefore is 5 times bigger than the $\beta_{\mathrm{c}}$ correction to the th-SZ. The maximum/minimum of $\Delta I_{\mathrm{D}, \mathrm{th}}^{\prime}$ is at $x=7.97 / 3.31$ and $\Delta I_{\mathrm{D}, \mathrm{th}}^{\prime}$ vanishes at $x=5.10(x=1$ corresponds to $v=$ $56.8 \mathrm{GHz}$ ). On the other hand the transformation of the kSZ leads to a y-type spectral distortion with the corresponding $y$-parameter $y_{\text {kin }}=\tau \beta_{\mathrm{c}, \|} \beta_{\mathrm{o}} \mu^{\prime} \sim 10^{-8} \mu^{\prime}$. The maximum of $\Delta I_{\mathrm{D}, \mathrm{k}}^{\prime}$ is at $x=5.10$. Figure 1 clearly shows that the motioninduced correction to the th-SZ easily reaches the level of a few percent in comparison to the k-SZ (e.g. at $v=400 \mathrm{GHz}$ it contributes $\sim 14 \%$ to the $\mathrm{k}-\mathrm{SZ}$ signal for a cluster with $k T_{\mathrm{e}}=$ $5.1 \mathrm{keV}$ and $\beta_{\mathrm{c}}=10^{-3}$ ).

In order to obtain the motion-induced change of the flux for unresolved clusters one has to integrate the surface brightness over the surface of the cluster. In the following we neglect the k-SZ, since its contribution only becomes important close to the crossover frequency. Then it follows that $\Delta F(v, \boldsymbol{n}) \propto \Delta I(v, \boldsymbol{n})$, implying that $\mathrm{d} \ln F / \mathrm{d} \ln v^{\prime}=\mathrm{d} \ln I / \mathrm{d} \ln x^{\prime}$. Comparing Eqs. (3b) and (12) one can define the effective spectral index of the SZ signal by

$\alpha=-\frac{\mathrm{d} \ln F\left(x^{\prime}\right)}{\mathrm{d} \ln x^{\prime}}=\frac{C_{\mathrm{th}}\left(x^{\prime}\right)}{Y_{0}\left(x^{\prime}\right)}-3$,

with $Y_{0}(x)=\mathcal{X}-4$. Using Eq. (14) one can write the CMB central brightness, flux and number count for unresolved clusters as

$$
\begin{aligned}
& I_{\mathrm{SZ}}^{\prime}\left(v^{\prime}, \boldsymbol{n}^{\prime}\right)=I_{\mathrm{SZ}}\left(v^{\prime}, \boldsymbol{n}^{\prime}\right)\left[1+\beta_{\mathrm{o}} \mu^{\prime}(3+\alpha)\right] \\
& \Delta F_{\mathrm{SZ}}^{\prime}\left(v^{\prime}, \boldsymbol{n}^{\prime}\right)=\Delta F_{\mathrm{SZ}}\left(v^{\prime}, \boldsymbol{n}^{\prime}\right)\left[1+\beta_{\mathrm{o}} \mu^{\prime}(1+\alpha)\right] \\
& \frac{\mathrm{d} N_{\mathrm{SZ}}^{\prime}}{\mathrm{d} \Omega^{\prime}}\left(F^{\prime}, \boldsymbol{n}^{\prime}\right)=\frac{\mathrm{d} N_{\mathrm{SZ}}}{\mathrm{d} \Omega}\left(F^{\prime}\right)\left[1+\beta_{\mathrm{o}} \mu^{\prime} \Sigma\right],
\end{aligned}
$$

with $\Sigma=2+\lambda(1+\alpha)$ and $\lambda=-\frac{\partial \ln \frac{\mathrm{d} N}{\mathrm{~d} \Omega}\left(F^{\prime}\right)}{\partial \ln F^{\prime}}$. Figure 2 shows the change of the central brightness and the flux for an unresolved cluster. It is obvious that only in the RJ region of the CMB spectrum the SZ brightness and flux do follow a powerlaw. The change of the number counts will be discussed below (see Sect. 4.1).

\section{Multi-frequency observations of clusters}

The observed CMB signal in the direction of a cluster consists of the sum of all the contributions mentioned above, including the relativistic correction to the SZ effect. Given a sufficient frequency coverage and spectral sensitivity one may in principle model the full signal for even one single cluster, but obviously there will be degeneracies which have to be treated, especially 
if noise and foregrounds are involved. Therefore it is important to make use of the special properties of each contribution to the total signal, such as their spectral features and spatial dependencies.

One obstacle for any multi-frequency observation of clusters is the cross-calibration of different frequency channels. Some method to solve this problem was discussed in Chluba \& Sunyaev (2004) using the spectral distortions induced by the superposition of blackbodies with different temperatures. In the following we assume that the achieved level of crosscalibration is sufficient. For more information on observational issues in the detection and analysis of the SZ effect see Birkinshaw \& Lancaster (2004).

The largest CMB signal in the direction of a cluster (after elimination of the CMB dipole) is due to the th-SZ. In order to handle this signal one can make use of the zeros of the spectral functions describing the relativistic corrections. In addition, future X-ray spectroscopy will allow us to accurately determine the mean temperature of the electrons inside clusters. This additional information will place useful constraints on the parameters describing the th-SZ and therefore may allow us to extract the effects connected to the peculiar velocities of the cluster and the observer.

The temperature difference related to the non-relativistic $\mathrm{k}-\mathrm{SZ}$ is frequency-independent and therefore may be eliminated by multi-frequency observations. As mentioned above (see Fig. 1) the motion-induced spectral distortion to the th-SZ has an amplitude and spectral dependence, which is very similar to the effect related to the first order $\beta_{\mathrm{c}}$ correction to the th-SZ. For many clusters on the other hand one can expect that the signals proportional to $\beta_{\mathrm{c}, \|}$ average out. This implies that for large cluster samples $\left(\sim 10^{3}-10^{4}\right)$ only the signals related to the th-SZ are important.

\subsection{Dipolar asymmetry in the number of observed clusters}

Integrating (15c) over solid angles leads to the observed number of clusters in a given region of the sky. If one assumes that the observed region is circular with radius $\theta_{\mathrm{r}}^{\prime}$ centered on the direction $\boldsymbol{n}_{0}^{\prime}=\left(\phi_{0}^{\prime}, \theta_{0}^{\prime}\right)$ then the total observed number of clusters is given by

$N_{\mathrm{SZ}}^{\prime}\left(F^{\prime}\right)=N_{\mathrm{SZ}, \mathrm{eff}}\left(F^{\prime}\right)\left[1+\beta_{\mathrm{o}} \mu_{0}^{\prime} \frac{1+\mu_{\mathrm{r}}^{\prime}}{2} \Sigma\right]$,

where $N_{\mathrm{SZ}, \text { eff }}\left(F^{\prime}\right)=4 \pi \frac{\mathrm{d} N_{\mathrm{SZ}}}{\mathrm{d} \Omega} \frac{1-\mu_{\mathrm{r}}^{\prime}}{2}$ is the effective number of clusters inside the observed patch with fluxes above $F^{\prime}, \mu_{\mathrm{r}}^{\prime}=$ $\cos \theta_{\mathrm{r}}^{\prime}$ and $\mu_{0}^{\prime}=\cos \theta_{0}^{\prime}$. For two equally sized patches in separate directions on the sky the difference in the number of observed clusters will then be

$\Delta N^{\prime}=\beta_{\mathrm{o}} N_{\mathrm{SZ}, \mathrm{eff}} \Delta \mu_{0}^{\prime} \frac{1+\mu_{\mathrm{r}}^{\prime}}{2} \Sigma$,

with $\Delta \mu_{0}^{\prime}=\mu_{0,1}^{\prime}-\mu_{0,2}^{\prime}$, where $\mu_{0, i}^{\prime}=\cos \theta_{0, i}^{\prime}$ for patch $i$. Centering the first patch on the maximum and the second on the minimum of the CMB dipole leads to the maximal change in the number of observed clusters at a given frequency $\left(\Delta \mu_{0}^{\prime}=2\right)$. To estimate the significance of this difference we compare $\Delta N^{\prime}$

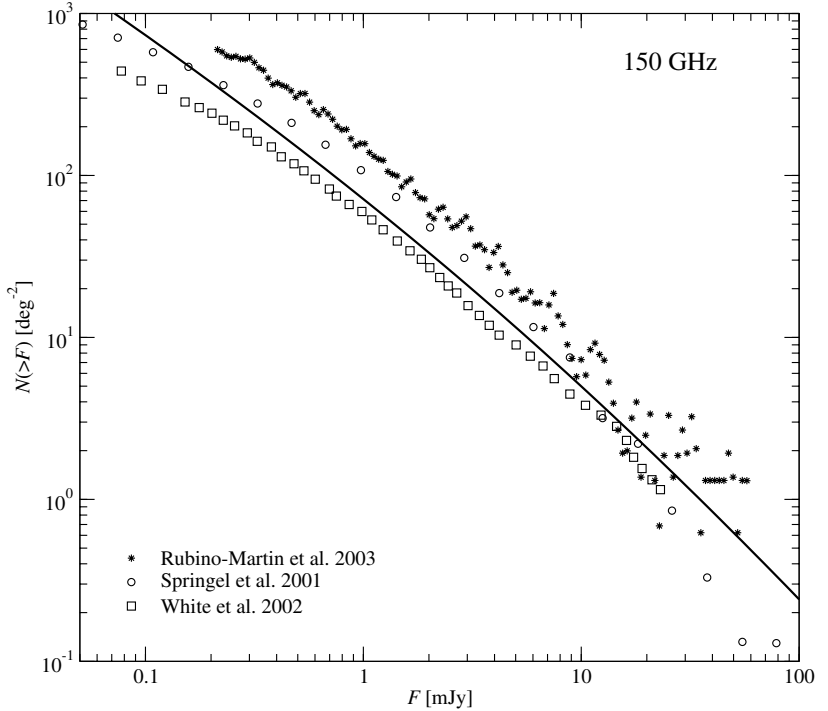

Fig. 3. $\log N-\log F$ : number of unresolved clusters per square degree with flux level above $F$ at observing frequency $v=150 \mathrm{GHz}$. The solid line shows the modified Press-Schechter prescription as used in this work.

to the Poissonian noise in the number of clusters for both patches, which is given by $\sqrt{N_{\mathrm{SZ}, 1}^{\prime}+N_{\mathrm{SZ}, 2}^{\prime}} \approx \sqrt{2 N_{\mathrm{SZ}, \mathrm{eff}}}$. To obtain a certain signal to noise level $q$ the inequality

$\beta_{\mathrm{o}}|\Sigma| \Delta \mu_{0}^{\prime}\left[1+\mu_{\mathrm{r}}^{\prime}\right] \sqrt{1-\mu_{\mathrm{r}}^{\prime}} \geq \frac{4 q}{\sqrt{N_{\mathrm{SZ}, \mathrm{f}}}}$

has to be fulfilled. We defined $N_{\mathrm{SZ}, \mathrm{f}}=4 \pi \frac{\mathrm{d} N_{\mathrm{SZ}}}{\mathrm{d} \Omega}$ as the number of clusters on the whole sky above a given flux level $F^{\prime}$. Here two effects are competing: the smaller the radius of each patch, the smaller the number of observed clusters above a given flux but the larger the effective $\left\langle\beta_{\mathrm{o}, \|}\right\rangle$. The optimal radius is $\theta_{\mathrm{r}} \sim 70^{\circ}$ but for a given $q$ and sensitivity the size in principle can be smaller.

\subsection{Numerical estimates for the dipolar asymmetry in the cluster number counts}

In this section we present results for the SZ cluster number counts using a simple Press-Schechter (Press \& Schechter 1974) prescription for the mass function of halos as modified by Sheth et al. (2001) to include the effects of ellipsoidal collapse. For simplicity we assume that all the clusters remain unresolved.

Since here we are interested in unresolved objects we only need to specify the cluster mass-temperature relation and baryonic fraction. For the former we apply the frequently used scaling relation and normalization as given by Bryan \& Norman (1998), whereas for the latter we simply assume a universal value of $\Omega_{\mathrm{b}} / \Omega_{\mathrm{m}}$, which is rather close to the local values as derived from X-ray data (e.g. Mohr et al. 1999) independent of cluster mass and redshift. We note that these two assumptions are the biggest source of uncertainty in our calculations and the use of them is only justified given the lack of current knowledge about the detailed evolution of the baryonic component in the Universe. In spite of these gross simplifications our results on cluster number counts agree very well with 


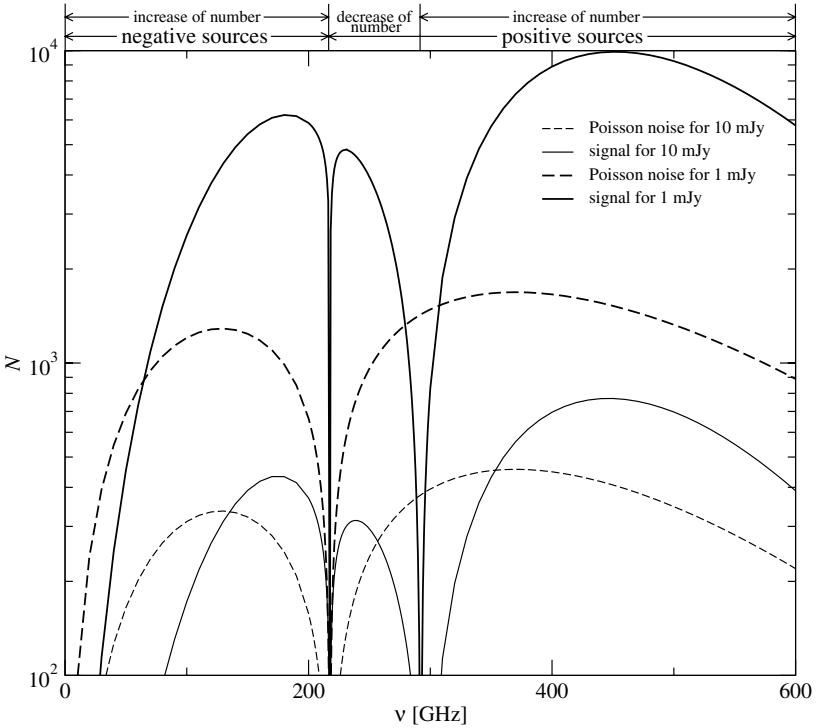

Fig. 4. Motion-induced dipolar asymmetry in number counts (solid lines) as a function of the observing frequency using the optimal patch radius $\theta_{\mathrm{r}}=70^{\circ}$ for both patches, where the first is centered on the maximum, the second on the minimum of the CMB dipole. For comparison we give the corresponding $1 \sigma$ Poissonian noise level (dashed curves). In addition we mark the regions where we expect an increase of the number of negative sources and a decrease/increase of the number of positive sources, respectively, if one is observing only in the direction of the maximum of the CMB dipole.

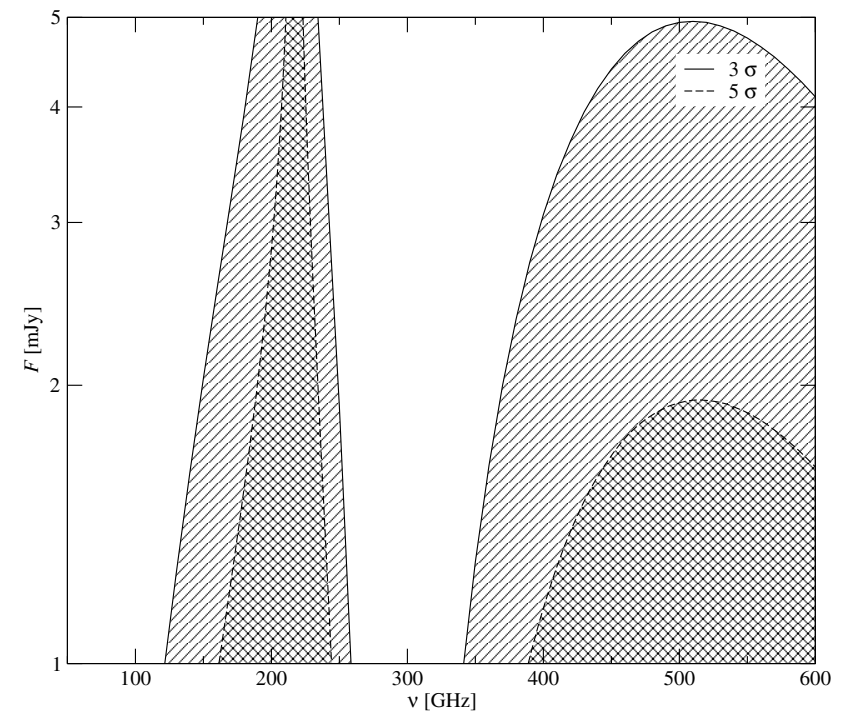

Fig. 5. Required sensitivities for a $3 \sigma$ and $5 \sigma$-detection of the motioninduced dipolar asymmetry in number counts as a function of frequency. The shaded areas indicate regions where a detection above $3 \sigma$ and $5 \sigma$ level is possible, respectively.

those obtained in state-of-the-art hydrodynamical simulations by Springel et al. (2001) and White et al. (2002) as demonstrated in Fig. 3. Here the counts are calculated for the $\Lambda \mathrm{CDM}$ concordance model (Spergel et al. 2003) assuming an observing frequency of $150 \mathrm{GHz}$. The first set of simulations included only adiabatic gas physics, whereas for the second also gas cooling and feedback from supernovae and galactic winds were taken into account. We also plot the results obtained by Rubiño-Martín \& Sunyaev (2003) using Monte-Carlo simulations based on a Press-Schechter approach. In the estimates presented below we will use the curve given by the solid line in Fig. 3, which in the most interesting range of lower flux limits $(1 \mathrm{mJy}-10 \mathrm{mJy})$ has an effective power-law slope in the range $\lambda \sim 1.10-1.25$.

In Fig. 4 we compare the motion-induced dipolar asymmetry in number counts as a function of the observing frequency using the optimal patch radius $\theta_{\mathrm{r}}=70^{\circ}$ for both sides of the sky with the $1 \sigma$ Poisson noise level for the two lower flux limits of $1 \mathrm{mJy}$ and $10 \mathrm{mJy}$. In addition we mark the regions where we expect an increase of the number of negative sources and a decrease/increase of the number of positive sources, respectively, if one is observing only in the direction of the maximum of the $\mathrm{CMB}$ dipole. It is important to note that the motion-induced change in the cluster number counts vanishes at frequencies $v \sim 300 \mathrm{GHz}$. The exact value of this frequency depends both on the slope of the number count curve and the spectral index.

Figure 5 presents the sensitivity limits where the motioninduced signal is equal to the $3 \sigma$ and $5 \sigma$ Poissonian noise levels for different observing frequencies. One can see that the most promising frequencies for a detection of the motioninduced asymmetries are around the crossover frequency (i.e. $\sim 217 \mathrm{GHz}$ ) and in the range $v \sim 400-500 \mathrm{GHz}$. Clearly, for a proper modeling near the crossover frequency one has to take into account the contribution from the k-SZ, which has been neglected so far. It is evident that the $\mathrm{k}-\mathrm{SZ}$ is contributing symmetrically to channels around $217 \mathrm{GHz}$ in the sense that the number of positive and negative sources is approximately equal. On the other hand in the range $v \sim 400-500 \mathrm{GHz}$ other astrophysical sources start to contribute to the source counts (see Sect. 4.3).

\subsection{Source count contribution from non-SZ populations}

In the range $v \sim 400-500 \mathrm{GHz}$, which is the most promising for a detection of the motion-induced number count asymmetry, other foreground sources begin to play a role, e.g. dusty high redshift galaxies (Blain et al. 2002). In the microwave band these galaxies have an extremely peculiar spectrum $F(v) \propto v^{-\alpha_{\mathrm{d}}}$, with $\alpha_{\mathrm{d}}$ ranging from -3 to -4 . Using formula (9) it is easy to show that the observed properties of this population will also be influenced by the motion of the Solar System, but in a completely different way than clusters: in the direction of our motion relative to the CMB rest frame their brightness and fluxes decrease when for clusters they increase. This implies that in the frequency range $v \sim 400-500 \mathrm{GHz}$ the motion-induced dipolar asymmetry in the number counts for these sources has the opposite sign in comparison to clusters, i.e. $\Delta N_{\mathrm{d}}^{\prime}<0$ when $\Delta N_{\mathrm{cl}}^{\prime}>0$. Detailed multi-frequency observations should allow us to distinguish the source count contributions of these two classes of objects, but nevertheless it is interesting that they have a different sign of the motioninduced flux dipole. 


\section{Conclusion}

We derived the changes to the SZ cluster brightness, flux and number counts induced by the motion of the Solar System with respect to the CMB rest frame. Since both the amplitude and direction of this motion are known with high precision it is easy to take these changes into account in the analysis and interpretation of future SZ data.

The corrections to the SZ cluster brightness and flux have a similar spectral dependence and amplitude as the first order velocity correction to the th-SZ (see Fig. 1). Since the motioninduced cluster signal can contribute $\sim 10 \%$ of the k-SZ it might be necessary to take it into account in future high precision CMB surveys, dedicated to accurately measuring the line of sight component of the cluster peculiar motions.

The dipolar asymmetry induced in the SZ cluster number counts in contrast to the counts of more conventional sources can change polarity depending on the observational frequency (see Sect. 4.3). This behavior is due to the very specific frequency dependence of the SZ effect. Our estimates show that frequencies around the crossover frequency $\sim 217 \mathrm{GHz}$ and in the range $\sim 400-500 \mathrm{GHz}$ are most promising for a detection of this motion-induced number count asymmetry (see Fig. 5).

Acknowledgements. We want to thank the anonymous referee for useful suggestions. Furthermore, we wish to thank M. Birkinshaw and S.H. Hansen for their comments on the manuscript. G.H. acknowledges the support provided through the European Community's Human Potential Programme under contract HPRN-CT-2002-00124, CMBNET, and the ESF grant 5347.

\section{References}

Baleisis, A., Lahav, O., Loan, A. J., \& Wall, J. V. 1998, MNRAS, 297, 545

Birkinshaw, M. 1999, Phys. Rep., 310, 97

Birkinshaw, M., \& Lancaster, K. 2004, Varenna Summer School review, available at

http://www.star.bris.ac.uk/ mb1/export.html
Blake, Ch., \& Wall, J. 2002, Nature, 416, 150

Blain, A. W., Smail, I., Ivison, R. J., Kneib, J. P., \& Frayer, D. T. 2002, Phys. Rep., 369, 111

Bryan, G. L., \& Norman, M. L. 1998, 495, 80

Challinor, A., \& Lasenby, A. 1998, ApJ, 499, 1

Challinor, A., \& van Leeuwen, F. 2002, Phys. Rev. D, 65, 103001, 1

Carlstrom, J. E., Holder, G. P., \& Reese, E. D. 2002, ARA\&A, 40, 643

Chluba, J., \& Sunyaev, R. A. 2004, A\&A, 424, 389

Church, S. 2002, technical report, scripts for talk available from http://ophelia.princeton. edu/ page/cmbpoltechnology-v2.ppt

Compton, A. H., \& Getting, I. A. 1935, Phys. Rev., 47, 817

Ellis, G. F. R., \& Baldwin, J. E. 1984, MNRAS, 206, 377

Fixsen, D. J., Cheng, E. S., Gales, J. M., et al. 1996, ApJ, 473, 576

Itoh, N., Kohyama, Y., \& Nozawa, S. 1998a, ApJ, 502, 7

Itoh, N., Kohyama, Y., \& Nozawa, S. 1998b, ApJ, 502, 17

Maoz, E. 1994, ApJ, 428, 454

Mohr, J. J., Mathiesen, B., \& Evrard, A. E. 1999, ApJ, 517, 627

Press, W. H., \& Schechter, P. 1974, ApJ, 187, 425

Rephaeli, Y. 1995a, ARA\&A, 33, 541

Rephaeli, Y. 1995b, ApJ, 445, 33

Rubiño-Martín, J. A., \& Sunyaev, R. A. 2003, MNRAS, 344, 1155

Sazonov, S. Y., \& Sunyaev, R. A. 1998, ApJ, 508, 1

Sazonov, S. Y., \& Sunyaev, R. A. 1999, MNRAS, 310, 765

Scharf, C. A., Jahoda, K., \& Boldt, E. 1995, ApJ, 454, 573

Sheth, R. K., Mo, H., \& Tormen, G. 2001, MNRAS, 323, 1

Smoot, G. F., Gorenstein, M. V., \& Muller, R. A. 1977, Phys. Rev. Lett., 39, 898

Spergel, D. N., Verde, L., Peiris, H. V., et al. 2003, ApJ, 148, 175

Springel, V., White, M., \& Hernquist, L. 2001, ApJ, 549, 681 (erratum $562,1086)$

Sunyaev, R. A., \& Zeldovich, Ya. B. 1972, Comments on Astrophysics and Space Physics, 4, 173

Sunyaev, R. A., \& Zeldovich, Ya. B. 1980a, ARA\&A, 18, 537

Sunyaev, R. A., \& Zeldovich, Ya. B. 1980b, MNRAS, 190, 413

Sunyaev, R. A., \& Zeldovich, Ya. B. 1981, Astrophys. Space Phys. Rev., 1,1

Strukov, I. A., Skulachev, D. P., Boyarskii, M. N., \& Tkachev, A. N. 1987, Soviet Astron. Lett., 13, 65

Zeldovich, Ya. B., \& Sunyaev, R. A. 1969, Ap\&SS, 4, 301

White, M., Hernquist, L., \& Springel, V. 2002, ApJ, 579, 16 